\title{
Large neurofibroma of the labia majora: A case report
}

\author{
HUSSEIN L. KIDANTO ${ }^{1,2,3^{*}}$, JOSHUA GARRISON ${ }^{1}$ and PETER WANGWE ${ }^{2}$ \\ ${ }^{1}$ Department of Obstetrics and Gynaecology, Muhimbili National Hospital, P.O. Box 65000, Dar es Salaam, Tanzania \\ ${ }^{2}$ Department of Obstetrics and Gynaecology, Muhimbili University of Health and Allied \\ Sciences, Dar es Salaam, Tanzania \\ ${ }^{3}$ Department of Women's and Children's Health, IMCH, Uppsala University, Uppsala, Sweden
}

\begin{abstract}
Neurofibromatosis is an autosomal dominant progressive disorder with an incidence of approximately 1 in 3000 live births. Its recognized features include hyper-pigmented skin lesions (cafe-au-lait spots), neurofibromas, iris hamartomas, macrocephaly, central nervous system tumours, defects of the skull and facial bones, and vascular lesions. Involvement of the external genitalia is extremely unusual. This report describes a case of a vulva neurofibroma in a 15-years old teenage girl with no history of trauma or features of Von Recklinghausen's disease. Treatment involved total excision of the tumour under spinal anaesthesia. The diagnosis of neurofibroma was confirmed by histological examination which showed spindle shaped cells with wavy nuclei arranged in a loose myxomatous stroma. No further treatment was offered but the patient was counselled on the possibility of recurrence. She was seen one month after excision and there were no signs of recurrence.
\end{abstract}

Keywords: vulva, labio majora, neurofibroma, Tanzania

\section{Introduction}

Vulva involvement by neurofibromas is found in about $18 \%$ of women with Von Recklinghausen's disease (Yükselet al., 2003). Although these tumours are usually small in size (less than $3 \mathrm{~cm}$ in diameter) and slow growing, giant rapidly growing solitary ones have been reported in the literature (Sa'adatu, 2006). Neurofibromas involving the female genital tract commonly involve the clitoris and the labia but may also affect the vagina, cervix endometrium, myometrium and ovary. They may be associated with urinary tract neruofibromatosis (Nicholas et al., 2009). Vulva neurofibromas have also been associated with trauma such as episiotomies or other vulva injuries (Yüksel, 2003).This report documents an unusual case of huge vulva neurofibroma that was managed at Muhimbili National hospital, a tertiary University teaching Hospital in Dar es Salaam, Tanzania.

\section{Case presentation}

A 15-year-old secondary school girl (grade three) presented to our gynaecology outpatient clinic on the $2^{\text {nd }}$ of June, 2011 complaining of a painless genital growth which gradually increased in size for one year, occasionally the growth was associated with itching and ulceration and mild pus-like discharge. There was no history of trauma or urinary symptoms. There was no swelling on any other part of her body and she had no family history of such swellings.

Physical examination revealed a globular pedunculated mass on the antero-lateral end of the right labium majora measuring about $20 \times 20 \mathrm{~cm}$ in size, there was some areas of infected ulcerations. It was a soft pedunculated mass, non-fluctuant, non-tender, translucent and was freely mobile. The pedicle was about $2.5 \mathrm{~cm}$ thick (Figure 1). The rest of the vulva was normal, hymen was intact and there were no swelling in other parts of the body. Full blood count was normal, and other blood

\footnotetext{
* Correspondence: Hussein L. Kidanto; E-mail: hkidanto@yahoo.co.uk
} 
investigations like electrolytes, urea, serum creatinine and liver function tests were all normal. The pelvic ultrasound examination did not reveal any pelvic abnormality.

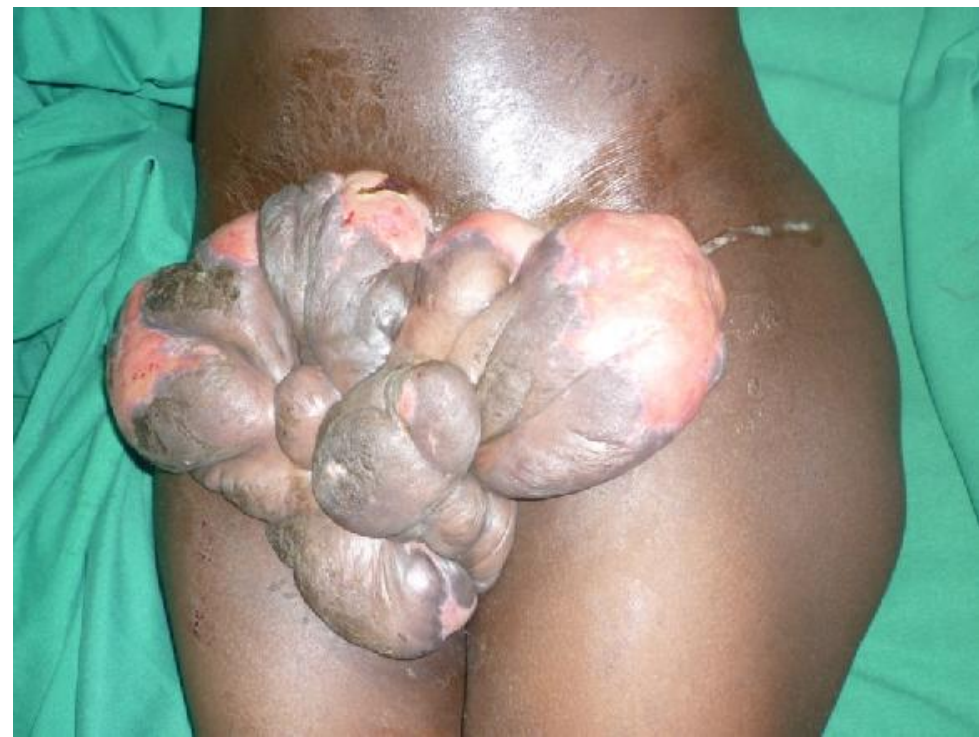

Figure 1: Large right labia majora neurofibroma with isolated ulceration, age 15 years

A provisional diagnosis of labial neurofibroma was reached based on clinical presentation. She was scheduled for excision biopsy. Total excision of the tumour was carried out under spinal anaesthesia. The diagnosis of neurofibroma was confirmed by histological examination which showed spindle shaped cells with wavy nuclei arranged in a loose myxomatous stroma containing some mast cells (Figure 2). No further treatment was offered but the patient was counselled on the possibility of recurrence. Patient was seen one month after excision and there were no signs of recurrence.

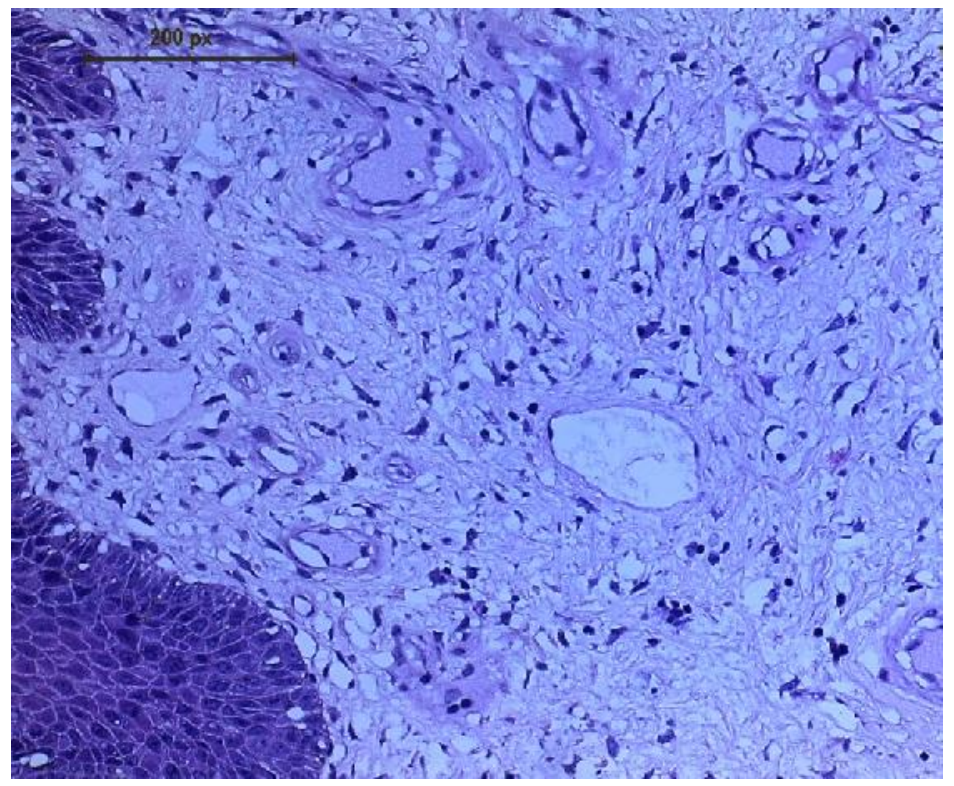

Figure 2: Histology results showing interlacing bundles of spindle shaped cells with wavy nuclei separated by loose myxomatous stroma 


\section{Discussion}

Neurofibroma is a rare benign tumour that rarely affects the vulva (Sa'adatu, 2006). Vulva involvement is found in about $18 \%$ of women with Von Recklinghausen's disease. This case report documents an unusual case of large labial neurofibroma affecting a teenage girl that was treated with simple total excision. Usually, these tumours are small in size (less than $3 \mathrm{~cm}$ in diameter) and slow growing. However, giant and rapidly growing solitary ones have been reported in the literature elsewhere (Venter, 1981). In this case the tumour was huge and it necessitated the patient to abandon school since she couldn't walk or dress properly. A previous report (Gordon et al., 1996; Yüksel, 2003) showed that these tumours can be a cause of intractable chronic pelvic pain as the case in this teenage girl. Generally, neurofibromas involving the female genital tract involve the clitoris and the labia but may also affect the vagina, cervix endometrium, myometrium, and ovary and may also be associated with urinary tract neruofibromatosis (Yüksel, 2003). This patient did not have any features of Von Recklinghausen's disease or involvement of other systems. She had no history of trauma although the association with vulva trauma and urinary tract neurofibromatosis should be borne in mind when managing these kinds of patients as it has been indicated in literature (Nicholas et al., 2009). Although the literature advocates a detailed examination of the genitourinary tract including cystoscopy, in this case we did not do cystoscopy since there was no symptom of urinary tract involvement from history and ultrasonography results.

The growth rate of this tumour was a bit rapid and the size was very large similar to cases reported in previously (Venter, 1981). However, rapid growth of any tumour is usually linked to malignant changes and thus it was important to rule out malignancy in this case, this was accomplished by thorough histological examination which found no features suggestive of malignant change. Since there were no areas suspicious of malignancy the excision biopsy was considered to be therapeutic. Patients who have had excision of such tumour should be followed-up closely because of possible recurrence.

After review of this case and the existing literature, we recommend that management of a labial neurofibroma should consist of total excision. We also recommend regular postoperative surveillance to monitor for local recurrence. The patient was closely followed-up in the Gynaecologic outpatient clinic and after three months there was no sign of recurrence. She is scheduled for further follow -up for one year.

\section{Conflict of interest}

None declared.

\section{Acknowledgments}

The authors would like to thank the Executive Director of Muhimbili National Hospital and the patients and her guardians for granting permission to publish this case report.

\section{References}

Gordon, M.D, Weilert, M. \& Ireland, K. (1996) Plexiform neurofibromatosis involving the uterine cervix, endometrium, myometrium and ovary. Obstetrics and Gynecology 88, 699-702.

Nicholas, G.C., Fabian, S., Arthur, G.W., Korgun, K. \& Linda, A.B. (2009) Neurofibromatosis presenting bas painless clitoromegaly. Journal of Urology 6, 220. 
Sa'adatu, T.S., Shehu, S.M. \& Umar, H.S. (2006) Neurofibroma of the labium majora: A case report. Nigerian Journal of Surgical Research 8, 99-100.

Venter, P.F., Röhm, G.F. \& Slabber, C.F. (1981) Giant neurofibromas of the labia. Obstetrics and Gynecology 57, 128-130.

Yüksel, H., Odabaşi, A.R., Kafkas, S., Onur, E. \& Turgut, M. (2003) Clitoromegaly in type 2 neurofibromatosis: a case report and review of the literature. European Journal of Gynaecological Oncology 24, 447-451. 